ISSN (print) 0867-2008 / ISSN (online) 239 I-75 I 2

DOI: http://dx.doi.org/10.12775/OM.2018.005

Klaus Militzer

Emeritus, Universität zu Köln

Winckelmannstr. 32

D-50825 Köln

Germany

klaus.militzer@uni-koeln.de

\title{
DIE EINGLIEDERUNG DER DEUTSCHORDENSBALLEI KOBLENZ IN DIE STRUKTUR DES KÖLNER ERZSTIFTS
}

\section{KEYWORDS}

history; the Middle Ages; military orders; Teutonic Order; Holy Roman Empire; ecclesiastical jurisdiction; Church administration; arbitration

\section{Abstract \\ The integration of the Koblenz bailiwick of the Teutonic Order in the structures of the Archbishopric of Cologne}

The chronological scope of the article covers the period from the first half of the I 3 th $\mathrm{c}$. to the mid- $5^{5}$ th $\mathrm{c}$., whereas the territorial scope - the commanderies of Koblenz, Cologne and Muffendorf. The research is based on the documents stored in the Historical Archive of the City of Cologne. The article tackles the issue of the independence of the Teutonic Order against the Archbishop of Cologne, who attempted to integrate the Teutonic Order with the structures of the Archbishopric of Cologne; however, in accordance with the papal privileges, the Teutonic Knights were subordinated to the Holy See. The paper consist of three parts which discuss three means of the policy of the Archbishop towards the Teutonic Knights. First, the means of such an integration was to include the official of the archbishop in the procedure of bestowal of the parishes to the priestbrothers. The presented candidate for the parish priest was obliged to take an oath to the Archbishop. Secondly, the Archbishop preferred to use his own jurisdiction over the papal jurisdiction. Thirdly, the Archbishop demanded payments from all the orders residing in his diocese. Despite these, the members of the Teutonic Orders could override these three means by addressing the Holy See directly. On the basis of this research, the author puts forward a thesis that the Teutonic Order was far less independent than it has been assummed so far. 
uf der siebten Konferenz der Ordines Militares. Colloquia Torunensia
Historica habe ich im Jahr I 993 über „Die Einbindung des Deutschen
Ordens in die süddeutsche Adelswelt" referiert. ${ }^{.}$Der vorliegende Fall geht weiter nördlich und betrifft den geistlichen Teil des Deutschen Ordens in Kurköln. Zunächst ist zu bemerken, dass die päpstliche Kurie und der Papst viele Fälle an sich zogen und dem Deutschen Orden zusicherten, dass er nur dem Papst und dessen Befehlsgewalt unterworfen sei. ${ }^{2}$ Aus der Fülle der überlieferten Papstbullen greifen wir nur einige wenige heraus, weil die abundante Überlieferung im vorliegenden Zusammenhang ein anderes Vorgehen nicht erlaubt. ${ }^{3}$ Am I6. Dezember 1220 bestätigte Papst Honorius III. dem Deutschen Orden unter anderen die Zehntfreiheit von allen Gütern, die der Orden selbst bewirtschafte. ${ }^{4}$ Dieselbe Urkunde wurde von Papst Gregor IX. I 227 und von Papst Alexander IV. I 259 und von Papst Urban IV. I 262 erneuert.' Beliebt war anfangs, die Deutschordenshäuser mit einem großen Gefolge zu belästigen. Auch das Vorgehen haben Päpste verboten, zunächst Honorius III. am I 6. Januar I 22 I. ${ }^{6}$ Die Bulle wiederholte I 244

I Vgl. Klaus Militzer, "Die Einbindung des Deutschen Ordens in die süddeutsche Adelswelt," in Ritterorden und Region. Politische, soziale und wirtschaftliche Verbindungen im Mittelalter, hrsg. v. Zenon Hubert Nowak, Ordines Militares. Colloquia Torunensia Historica VIII (Toruń: Wydawnictwo Uniwersytetu Mikołaja Kopernika, I995), I4I-I60; Wiederabdruck in: Klaus Militzer, Zentrale und Region. Gesammelte Beiträge zur Geschichte des Deutschen Ordens in Preussen, Livland und im Deutschen Reich aus den Jahren 1968 bis 2008, Quellen und Studien zur Geschichte des Deutschen Ordens $75=$ Veröffentlichungen der Internationalen Historischen Kommission zur Erforschung des Deutschen Ordens I 3 (Weimar: vdg, 201 5 ), 322-34I; vgl. auch neuerdings für andere Länder: Kristjan Toomaspoeg, "Der Verlust der Besitzungen des Deutschen Ordens in Italien am Ende des I 5. und zu Beginn des I 6. Jahrhunderts," Ordines Militares Colloquia Torunensia Historica. Yearbook for the Study of the Military Orders 16 (201 I): I 35, I 37; Marie-Luise Favreau-Lilie, "Reformbedarf und Reformen an der norditalienischen Peripherie. Der Deutsche Orden in Venedig und in Venetien (I 4.- I 5. Jh.)," Ordines Militares Colloquia Torunensia Historica. Yearbook for the Study of the Military Orders I 6 (20 I I): 80-8 I.

2 Vgl. allgemein: Hans Erich Feine, Kirchliche Rechtsgeschichte. Katholische Kirche (Köln-Graz: Böhlau, 1964, 4. erweiterte Aufl.), 35 I-63; Wilhelm Janssen, Das Erzbistum Köln im späten Mittelalter, IIgI-ISIS, Tl. I, Geschichte des Erzbistums Köln 2 (Köln: Bachem, I995), I I 3 $-\mathrm{I} 20$.

3 Im Verlauf der Arbeit wird noch auf andere Papstbullen hingewiesen.

4 Tabulae Ordinis Theutonici, hrsg. v. Ernst Strehlke (Berlin: Weidmann, I 869. Neudruck: Toronto: Prelum Academicum Universitatis Torontonensis, I 869), Nr. 306; Die Urkunden des Deutschordenszentralarchivs in Wien. Regesten, Tlbd. I, II 22 - Januar I3I3, hrsg. v. Udo Arnold, Quellen und Studien zur Geschichte des Deutschen Ordens 60/I = Veröffentlichungen der Internationalen Historischen Kommission zur Erforschung des Deutschen Ordens I I I (Marburg: N. G. Elwert, 2006), Nr. 54.

5 Die Urkunden, I, hrsg. v. Arnold, Nrn. I 7 I, 528, 588.

6 Tabulae, hrsg. v. Strehlke, Nr. 3 I 7 ; Die Urkunden, I, hrsg. v. Arnold, Nr. 68. 
und I 247 Papst Innozenz IV.7 Am I 9. Januar 122 I verbot Papst Honorius III. allen geistlichen Reisenden, den Zehnten oder andere Abgaben von den Deutschordenshäusern zu verlangen. ${ }^{8}$ Dieselbe Bulle bekräftigte Papst Innozenz IV. in den Jahren I 244 und I 247. ${ }^{9}$ Die Nachweise ließen sich fortsetzen, bis eigentlich erst unter Papst Martin V. ein Wandel eintrat. Einmal bestimmten dieser Papst und seine Nachfolger, dass alle Privilegien vorangegangener Päpste gültig seien. ${ }^{10}$ Ferner verfügten sie allgemein, dass sie hochrangige geistliche Personen, die den Ereignissen näher standen, mit der Aufgabe der Überwachung der Privilegien und deren Durchführung beauftragten.

Immerhin stellten Päpste seit dem Ende des I 2. Jahrhunderts immer wieder Bullen aus, die den Deutschen Orden mit den beiden älteren Ritterorden der Templer und Johanniter gleichstellten und der Jurisdiktion des Papstes unterwarfen. ${ }^{11}$ Besonders hervor tat sich Papst Honorius III. in den Jahren ab i 2 I 6 und besonders im Jahr I 22 I. ${ }^{12}$ Dadurch, dass die Päpste und deren Kurie immer mehr Befugnisse an sich zogen, verringerten sie zumindest die Möglichkeit eines Eingriffs des Kölner Metropoliten in diese Bereiche und ebenso in die Verwaltung des Deutschen Ordens. ${ }^{13}$

Allerdings führten die Kölner Erzbischöfe nicht immer die Befehle der Päpste aus und verweigerten beispielsweise unter dem Erzbischof Friedrich von Saarwerden die Bezahlung der schuldigen Servitien oder Annaten. ${ }^{14}$ Jedoch blieb das Vorgehen eine Ausnahme.

Die Deutschordenskommenden Köln, Rheinberg, Judenrode und Muffendorf lagen in der Diözese Köln, die Kommenden Koblenz, Mecheln und Ibersheim dagegen in fremden Diözesen, nämlich denen von Trier, Utrecht und

7 Die Urkunden, I, hrsg. v. Arnold, Nrn. 284, 317.

8 Tabulae, hrsg. v. Strehlke, Nr. 332; Die Urkunden, I, hrsg. v. Arnold, Nr. 78.

9 Die Urkunden, I, hrsg. v. Arnold Nrn. 279, 325.

1o Tabulae, hrsg. v. Strehlke, Nrn. 706, 7 I 8, 72 1, 723.

II Tabulae, hrsg. v. Strehlke, 263-47 I; Udo Arnold, "Der Deutsche Orden zwischen Kaiser und Papst im I 3. Jahrhundert," in Ritterorden zwischen geistlicher und weltlicher Macht, hrsg. v. Zenon Hubert Nowak, Ordines Militares. Colloquia Torunensia Historica V (Toruń: Wydawnictwo Uniwersytetu Mikołaja Kopernika, I 990), 59-60.

I2 Tabulae, hrsg. v. Strehlke, Nrn. 304-364 und weitere Bullen: Die Urkunden, I, hrsg. v. Arnold, Nrn. 32-33, 40, 54-55, 56, 58-100 und öfter.

13 Feine, Kirchliche Rechtsgeschichte, 355-356; Klaus Militzer, Von Akkon zur Marienburg. Verfassung, Verwaltung und Sozialstruktur der Deutschen Ordens II90-I309, Quellen und Studien zur Geschichte des Deutschen Ordens $56=$ Veröffentlichungen der Internationalen Historischen Kommission zur Erforschung des Deutschen Ordens 9 (Marburg: N. G. Elwert, I 999), I 9.

I4 Insbesondere Janssen, Erzbistum, I: I I 5- I I 6. 
Worms. ${ }^{15}$ Wir konzentrieren uns in den folgenden Ausführungen auf die Kommende Köln, da nun die Regesten aus dem Archiv der Kölner Kommende herausgegeben worden sind. ${ }^{16}$

Ein Einfallstor für die Eingliederungen des Deutschen Ordens in die einzelnen Bistümer oder Erzbistümer waren in erster Linie die Offiziale. In Köln gab es mehrere derartige Amtsträger, nämlich einmal den erzbischöflichen Offizial, der gewöhnlich im Saalbau im Süden des Kölner Doms tagte, den des Propstes und den des Dekans des jeweiligen Kölner Domkapitels. Daneben waren noch die Offiziale des Bonner Propstes, die vorwiegend in Bonn, und des Xantener Propstes, die vorwiegend in Xanten Recht sprachen und tätig wurden. ${ }^{17}$ Ferner haben andere Geistliche in hervorragenden Ämtern wenigstens im I 3. Jahrhundert und darüber hinaus Offiziale unterhalten oder eingesetzt. ${ }^{18}$

Der Offizial des Kölner Erzbischofs ist erstmals zum Jahr I 252 bezeugt. Es war der Scholaster von St. Severin namens Andreas. ${ }^{19}$ Die Offiziale des Erzbischofs waren nicht immer in Köln ansässig, sondern schlichteten auch außerhalb der Stadt Streitigkeiten mit dem Deutschen Orden. So war der Offizial im Jahr I 293 in Neuss im Haus des Pfarrers Lambert tätig geworden. ${ }^{20}$ Aber das blieb eine Ausnahme. Der Kölner Offizial oder der Offizial des Kölner Erzbischofs tagte seit dem I 4. Jahrhundert jedenfalls vorwiegend im Kölner Saal südlich des Doms. Erst im I 4. Jahrhundert kamen die Offiziale der Pröpste und Dekane des Domstifts und der Pröpste von Bonn und Xanten hinzu. Bedeutender für die Angelegenheiten des Ordens blieb jedoch der im Saal tagende Offizial des Erzbischofs.

is Vgl. Hans Limburg, Die Hochmeister des Deutschen Ordens und die Ballei Koblenz, Quellen und Studien zur Geschichte des Deutschen Ordens 8 (Bad Godesberg: Verlag Wissenschaftliches Archiv, 1969), I6-17, und öfter.

${ }^{16}$ Die Urkunden der Deutschordenskommende St. Katharinen zu Köln. Regesten, Tlbd. I, Juni I218 - November 1496, hrsg. v. Klaus Militzer, Quellen und Studien zur Geschichte des Deutschen Ordens 78/I-II = Veröffentlichungen der Internationalen Historischen Kommission zur Erforschung des Deutschen Ordens I6/I-II (Weimar: Verlag und Datenbank für Geisteswissenschaften, 2016).

17 Janssen, Erzbistum, I: 338-355; Franz Gescher, "Das Offizialat der Erzbischöfe von Köln im I 3. Jahrhundert," Annalen des Historischen Vereins für den Niederrhein I I 5 ( 1929): I $36-$ I66; ders., "Die erzbischöfliche Kurie von ihren ersten Anfängen bis zur Gegenwart, Eine rechtsgeschichtliche Skizze," Annalen des Historischen Vereins für den Niederrhein I I 8 ( I 93 I): I - 3 I.

18 Die Urkunden, I, hrsg. v. Militzer, Nr. I 30. Am I9. Februar I 288 ist ein Offizial des Propstes von St. Kunibert in Köln bezeugt. Derartige Offiziale können aber vernachlässigt werden.

19 Janssen, Erzbistum, I : 339; Gescher, "Das Offizialat," I 37-I 38; vgl. auch Die Urkunden, I, hrsg. v. Militzer, Nr. 29. Möglicherweise war Andreas schon ein paar Jahre früher Offizial: Gescher, "Das Offizialat," I 4 I.

20 Die Urkunden, I, hrsg. v. Militzer, Nr. I 48. 
Nun haben die Offiziale immer wieder Urkunden bestätigt oder Streitigkeiten geschlichtet, auch zu den Streitfällen ihre Siegel zur höheren Glaubwürdigkeit an den Urkunden angebracht. Aber sie nahmen auch, vermehrt seit dem I 4. Jahrhundert, Präsentationen von Priestern für bestimmte Pfarreien entgegen und überprüften diese Personen auf ihre Eignung. Alle diese Tätigkeiten der Offiziale waren mit Gebühren verbunden und mussten bezahlt werden.

Schon Papst Honorius III. hatte am I 8. Januar I 22 I dem Deutschen Orden das Privileg verliehen, alle dem Bischof präsentierten tauglichen Priester für die vorgesehenen Pfarreien zuzulassen. ${ }^{21}$ Am 22. Mai 1237 erteilte Papst Gregor IX. dem Orden eine Bulle, dass die Bischöfe oder deren Beauftragte, die Priester, die der Orden präsentiere, ohne weiteres in die dem Orden zustehenden Patronatskirchen einzusetzen hätten. ${ }^{22}$ Ferner gestattete Papst Alexander IV. dem Orden, auch Weltpriester für die Patronatskirchen zu präsentieren. ${ }^{23}$ Die Privilegien wurden immer wieder erneuert oder transsumiert oder auch neue, ähnlich klingende Bullen erworben, weil sie dem Orden wichtig waren.

Am 5. Februar I 49 I wurde in einem Notariatsinstrument ausführlich dargelegt, was unter einer vollständigen Investitur der Patronatskirche von Hackenbroich zu verstehen sei. Die Pfarrkirche von Hackenbroich gehörte zur Ballei Koblenz. ${ }^{24}$ Infolgedessen wird der Komtur oder Landkomtur von Koblenz den Pfarrer präsentiert haben. Nach dem genannten Bericht zeigte ein Notar die besiegelte Urkunde des Offizials vor, führte den angehenden Pfarrer in die Kirche, die er durch jenen aufschließen ließ, ging mit ihm zum Altar und zeigte ihm das Messbuch, den Kelch, das Missale und anderes Zubehör, das der Kandidat wohl auch berührte. Ferner ließ der Notar den angehenden Pastor das Glockenseil anfassen und wohl auch die Glocken läuten, und wies auf das Taufbecken in einem abgesonderten Teil der Kirche hin. Anschließend ging er mit dem Priester zum Pfarrhaus und ließ ihn davon Besitz ergreifen. ${ }^{25}$ Aber das war noch nicht alles. Wenn der Koblenzer Komtur einen Priesterbruder für gewöhnlich einem Offizial präsentierte, hatte der Offizial oder dessen Beauftragter die Gemeinde einer Pfarrkirche dreimal von der Kanzel an Sonn- und Feiertagen zu fragen, ob Widerspruch

${ }_{21}$ Tabulae, hrsg. v. Strehlke, Nr. 327; Die Urkunden, I, hrsg. v. Arnold, Nr. 72, mit weiteren Nachweisen.

22 Tabulae, hrsg. v. Strehlke, Nr. 466; Die Urkunden, I, hrsg. v. Arnold, Nr. 243, mit weiteren Nachweisen.

23 Tabulae, hrsg. v. Strehlke, Nr. 58 I; Die Urkunden, I, hrsg. v. Arnold, Nr. 492.

24 Limburg, Die Hochmeister, 25; Klaus van Eickels, Die Deutschordensballei Koblenz und ibre wirtschaftliche Entwicklung im Spätmittelalter, Quellen und Studien zur Geschichte des Deutschen Ordens 52 (Marburg: N. G. Elwert, I 995), I 82-1 83, 272.

25 Die Urkunden, I, hrsg. v. Militzer, Nr. 932. 
erhoben werde. Meist wurde solch ein eventueller Widerspruch vor dem Offizial in Köln vorgetragen. Wenn die dreimalige Ausrufung geschehen war oder auch schon vorher, hatte der Kandidat zu schwören - meist mit einer Hand auf dem Evangelium -, dem Papst, dem Kölner Erzbischof, dem Archidiakon und dem Offizial gehorsam zu sein. Dann setzte der Offizial dem knieenden Kandidaten das Barett oder Birett auf den Kopf und nahm ihm das Versprechen ab, für das Seelenheil seiner Pfarrkinder und die in der Kirche aufbewahrten Reliquien zu sorgen. ${ }^{26}$ Es kam auch vor, dass andere Formalien beobachtet wurden, beispielsweise investierte der Offizial im Jahr I 39 I einen Kandidaten mit einem Buch, ${ }^{27}$ gemeint ist wohl das Messbuch. Es ist auch nachweisbar, dass ein Erzbischof ausnahmsweise die Investitur eines Ordensbruders selbst wahrnahm ${ }^{28}$ oder dass ein Vertreter des Papstes sie vornahm. ${ }^{29}$ Wenn also meist der Offizial des Erzbischofs, des Propstes oder des Dekans des Domstiftes den Kandidaten prüften oder dreimal in der entsprechenden Kirche ausrufen ließen, waren sie im allgemeinen nicht persönlich zur Stelle, sondern beauftragten näher gelegene Pfarrer damit. Diese Pfarrer entledigten sich der Aufgabe und hefteten Transfixe an die Auftragsurkunde. ${ }^{30}$ Auf diese Art und Weise blieb dem Offizial eine vielfach unbequeme Reise zu den einzelnen Pfarreien erspart. Allerdings hatte er auch nicht die Zeit, derartige Aufgaben zu erfüllen.

Das Vorgehen betraf nicht nur Pfarrer und Priesterbrüder des Deutschen Ordens, sondern in gleicher Weise auch Weltpriester, Vikare und Altaristen, die nicht unbedingt dem Orden angehörten. Jedoch ist es nicht in allen Fällen so gewesen, dass auch Altaristen, Vikare oder Ewigvikare den Offizialen zugeführt wurden. Für einen derartigen Nachweis liefern die Urkunden und Akten für das Mittelalter keinen Anlass.

Die Präsentation zumindest der Priester vor dem Offizial oder gar dem Erzbischof war keineswegs, wie schon gesagt, unentgeltlich. Es wurden vielmehr Gebühren erhoben, die zunächst den Bedarf des Offizials decken sollten, aber nicht selten darüber hinaus gingen. ${ }^{31}$ Die Präsentation und die entsprechende Gebühr stellten jedenfalls ein Mittel dar, den Orden in die kirchliche Organisation des Erzbistums einzugliedern.

26 Ebd., Nrn. 275, 280.

27 Ebd., Nrn. 563, 564

28 Ebd., Nr. 309; Regesten der Erzbischöfe von Köln im Mittelalter, Bd. 4, 1304-1332, bearb. v. Wilhelm Kisky (Düsseldorf-Bonn: Droste, Hanstein, I 9 I 5 ), Nr. I 949.

29 Die Urkunden, I, hrsg. v. Militzer, Nrn. 3 I o-3 I I. Das kam häufiger vor: ebd., Nr. I 4 I ( 1292 ); Nr. 3 I 4 ( 1332 ) und öfter.

30 Beispielsweise 1392 an die Urkunde von I 39 I: Die Urkunden, I, hrsg. v. Militzer, Nrn. 567-568.

${ }_{31}$ Vgl. Janssen, Erzbistum, I: 346. 
Das zweite Einfallstor für den Kölner Erzbischof, den Orden in die kirchliche Organisation einzugliedern und nicht nur für ihn, war die Gerichtsbarkeit. Der Deutsche Orden war allerdings generell der päpstlichen Gerichtsbarkeit unterworfen und der Gerichtsbarkeit der Bischöfe und Erzbischöfe enthoben. Schon im Jahr I I 9 I nahm Papst Clemens III. den neuen Orden in seinen Schutz. ${ }^{32}$ Seine Nachfolger haben immer wieder betont, dass der Orden und auch deren Angehörige direkt vor dem Papst oder dessen Bevollmächtigten Klage erheben könnten. ${ }^{33}$ Ausdrücklich ist die Tatsache im Jahr 1319 erwähnt. ${ }^{34}$ Es ist klar, dass der Papst und die Kurie nicht alle Streitigkeiten schlichten konnten. Sie delegierten daher zumindest seit der Mitte des I 3. Jahrhunderts ihre Gerichtsgewalt an Prälaten, die nicht ohne weiteres mit der in Rede stehenden Tat in Verbindung standen, aber ihren Schwerpunkt dennoch geographisch nahe genug am Ort der Tat hatten. Im Jahr I 257 trug Papst Alexander IV. beispielsweise dem Abt von St. Alban auf, den Mainzer Erzbischof und die Prälaten der Umgebung zu überzeugen, dass sie den Deutschen Orden nicht mit Abgaben belasten sollten. ${ }^{35}$ Im Jahr I 258 machte derselbe Papst den Dekan von St. German in Speyer zu seinem Bevollmächtigten. ${ }^{36}$ Im Jahr 1275 ernannte der Papst den Dekan von St. Florin in Koblenz zum Konservator des Ordens in Deutschland. ${ }^{37}$ Seitdem haben Päpste immer wieder Prälaten zu Konservatoren erhoben oder mit einer Streitschlichtung beauftragt. ${ }^{38} \mathrm{Im}$ Jahr 1292 hatte der Kantor von St. Florin in Koblenz, der vom Dekan von St. Mariengraden in Köln unterdelegiert worden war. Er hat seinerseits Pfarrer mit der Verfolgung einer Klage beauftragt. ${ }^{39}$ In Laufe des Mittelalters wurden zunehmend Bischöfe und Erzbischöfe mit dem Konservatorenamt betraut, aber die Päpste handhabten die Aufgaben nicht immer so.

Auch wenn nicht alle Streitsachen in Rom an der Kurie entschieden worden sind, stand es dem Deutschen Orden oder dessen Angehörigen doch frei, ein Urteil des Papstes einzuholen. Viele Entscheidungen, die geographisch näheren Prä-

32 Tabulae, hrsg. v. Strehlke, Nr. 295; Die Urkunden, I, hrsg. v. Arnold, Nr. 7.

33 Vgl. beispielsweise: Tabulae, hrsg. v. Strehlke, Nrn. 305-306, 3 I I Die Urkunden, I, hrsg. v. Arnold, Nrn. 40, 54, 58 und öfter.

34 Die Urkunden des Deutschordenszentralarchivs in Wien. Regesten, Tlbd. II, Februar 1313 - November ${ }_{14} 18$, hrsg. v. Udo Arnold, Quellen und Studien zur Geschichte des Deutschen Ordens $60 / \mathrm{II}=$ Veröffentlichungen der Internationalen Historischen Kommission zur Erforschung des Deutschen Ordens i I/II (Marburg: N. G. Elwert, 2007), Nr. I 425.

35 Die Urkunden, I, hrsg. v. Arnold, Nr. 470.

36 Tabulae, hrsg. v. Strehlke, Nr. 592; Die Urkunden, I, hrsg. v. Arnold, Nr. 508.

37 Die Urkunden, I, hrsg. v. Arnold, Nr. 763.

38 Vgl. zum Beispiel: Die Urkunden, I, hrsg. v. Arnold, Nrn. 86 I, 868, 875, 877, 903, 907-908, 9 I 3 und öfter.

39 Die Urkunden, I, hrsg. v. Militzer, Nr. I 4 I. 
laten, Bischöfen oder Erzbischöfen überlassen wurden, waren vorher an der päpstlichen Kurie anhängig gemacht worden. Dabei konnte es sich auch um Pfründen von Priesterbrüdern handeln, die nicht in deren Sinne entschieden worden sind. Ein Beispiel ist der Kölner oder Koblenzer Priesterbruder Adam Haer aus Jülich. Er prozessierte am Ende des I 5 . Jahrhunderts am päpstlichen Hof in Rom um die Stelle eines Pfarrers in Elsen. Diese Pfründe verweigerte ihm Werner Overstolz, der Komtur von Koblenz..$^{4}$ Also klagte der Priesterbruder vor dem Papst. Das Urteil überließ das katholische Oberhaupt aber einem geographisch näher stehenden Prälaten. Das war dieses Mal im Jahr I 482 Johann von Arsen, der Propst an der Heilig-Geist-Kirche in Roermond, der aber in Köln lebte. Er forderte Gehorsam von allen gegenüber seinem gefällten Urteil - eben kraft päpstlicher Autorität. Der Komtur von Koblenz beugte sich dem Richterspruch und präsentierte den Ordenspriester Adam Haer auf die Stelle eines Pfarrers von Elsen. Zumindest seit 1498 ist er als Pfarrer von Elsen belegt. ${ }^{41}$

Zum dritten ist zu erwähnen, dass Kölner Erzbischöfe einen Streit zwischen dem Deutschen Orden und einem geistlichen Untergebenen, einem Stift oder Kloster zu schlichten versuchten, und es auch taten. So entschieden sie beispielsweise eine Auseinandersetzung in den Jahren I 2 I 9 oder 1220 zwischen dem Deutschen Orden und dem Stift St. Severin in Köln mit Hilfe von Herren des Stifts St. Florin in Koblenz. Danach sollte neben anderen der Orden verpflichtet sein, dem Kustos des Severinsstifts 20 Mark Kölnisch zu überreichen. Allein schon die Streitschlichtung bestimmte den Orden, Geld zu zahlen, ${ }^{42}$ die auch bei Nichterfüllung der anderen Pflichten erweitert werden oder andere Maßnahmen nach sich ziehen konnten. Die unterschiedliche Datierung, die offenbar auf Abschriften zurückgeht, ${ }^{43}$ braucht uns nicht zu interessieren. Wichtig bleibt vielmehr die Aussage, dass das Kölner Stift St. Severin nur unter besonderen Bedingungen bereit war, auf seine Rechte zu verzichten. Allerdings hat sich im Laufe der Zeit diese Verpflichtung der Deutschordenskommende verflüchtigt. Sie wurde jedenfalls

$40 \mathrm{Zu}$ ihm: Limburg, Die Hochmeister, I09-1 25. Allerdings geht er auf den Streit nicht ein.

${ }^{41}$ Regesta historico-diplomatica Ordinis S. Mariae Theutonicorum, Tl. I, Index Tabularii Ordinis S. Mariae Theutonicorum / Regesten zum Ordensbriefarchiv, bearb. unter Mitwirkung zahlreicher Anderer v. Erich Joachim, hrsg. v. Walther Hubatsch, Bd. 2, I455-ISIo (Göttingen: Vandenhoeck \& Ruprecht, I950), Nr. I 8039.

${ }_{42}$ Regesten der Erzbischöfe von Köln im Mittelalter, Bd. 3, I205-I304, Hälfte I, I205-I26I, bearb. v. Richard Knipping (Düsseldorf-Bonn: Droste, Hanstein, 1909), Nr. 230, mit dem Hinweis, dass in diesem Fall kein Osterstil zu verzeichnen sei. Anders auf I 220 Jan. 26 datiert: Die Urkunden, I, hrsg. v. Arnold, Nr. 5 I; Codex diplomaticus ordinis sanctae Mariae Theutonicorum. Urkundenbuch des Deutschen Ordens, hrsg. v. Johannes Heinrich Hennes, Bd. 2 (Mainz: Kirchheim, I 86I ), Nr. I 2; vgl. van Eickels, Die Deutschordensballei Koblenz, 3 I 6 Nr. 2004.

43 Vgl. die Ausführungen von Karin Jedner auf dieser Konferenz. 
nicht wiederholt. Diese Art der Streitschlichtung blieb jedoch kein Einzelfall, sondern wiederholte sich immer wieder. ${ }^{44}$

Seit dem I3. Jahrhundert hatte die Schiedsgerichtsbarkeit der Kölner Erzbischöfe einen Aufschwung genommen. ${ }^{45}$ Ihm konnte sich der Deutsche Orden trotz der päpstlichen Vorbehalte nicht entziehen. Wenn beispielsweise ein Konkurrent des Ordens den Kölner Erzbischof ins Spiel brachte, konnten der Deutsche Orden und der Koblenzer Komtur dem kaum etwas entgegensetzen. Wilhelm Janssen selbst hat die Schiedsgerichtsbarkeit anhand der Auseinandersetzungen des Ordens um das Neusser Spital von 1250 herangezogen. ${ }^{46}$ Das Einwirken eines Mächtigen - als solcher ist der Kölner Erzbischof zu betrachten - in einen Bereich, der ihm sonst entzogen war, blieb das Bestreben des Metropoliten. ${ }^{47}$ Er hat eine solche Schiedsgerichtsbarkeit gegenüber dem Deutschen Orden auch benutzt.

Im Jahr I 285 befreite Erzbischof Siegfried von Westerburg die Komture von Koblenz und Ramersdorf von allen Abgaben, insbesondere den Rheinzöllen, wegen ihrer geleisteten Dienste..$^{4}$ Ferner quittierte er beiden Komturen den Betrag von 400 Mark Kölnisch, da sie Bürgen für die verstorbene Gräfin von Sayn geworden seien. ${ }^{49}$ Zudem verbot der Papst in den Jahren I 448 und I 449 die Anwendung von Urteilen fremder Gerichte über Einkünfte, Ländereien und Personen des Deutschen Ordens..$^{50}$ Zwar erteilte der Papst das Verbot eher dem Zugriff der

44 So 1389 , vgl. Die Urkunden, I, hrsg. v. Militzer, Nr. 557; Regesten der Erzbischöfe von Köln im Mittelalter, Bd. 9, I38I-I390 (Friedrich von Saarwerden), bearb. v. Norbert Andernach (Düsseldorf: Droste, I 983 ), Nr. I 83 I, oder allgemein: Die Urkunden, I, hrsg. v. Arnold, Nr. 368.

45 Dazu Wilhelm Janssen, "Bemerkungen zum Aufkommen der Schiedsgerichtsbarkeit am Niederrhein im I 3. Jahrhundert," Jahrbuch des Kölnischen Geschichtsvereins 43, H. I ( 97 I ): 77 und öfter.

${ }^{46}$ Urkundenbuch für die Geschichte des Niederrheins oder des Erzstifts Cöln, der Fürstenthümer Jülich und Berg, Geldern, Meurs, Cleve und Mark, und der Reichsstifte Elten, Essen und Werden, Bd. 2, Von dem Jahr I20I bis I3oo einschliesslich, hrsg. v. Theodor Joseph Lacomblet (Düsseldorf: J. Wolf, I 846), Nr. 358; Regesten der Erzbischöfe, 3.I, bearb. v. Knipping, Nr. I 578; Die Urkunden, I, hrsg. v. Militzer, Nrn. 25-27. Im Archiv der Kommende Köln sind drei Ausfertigungen erhalten geblieben.

47 Janssen, "Bemerkungen," 77-85.

48 Regesten der Erzbischöfe von Köln im Mittelalter, Bd. 3, I205-I305, Hälfte 2, I26I-I304 und Register zu beiden Hälften, bearb. v. Richard Knipping (Düsseldorf-Bonn: Droste, Hanstein, I9I3), Nr. 3079; Die Deutschordensurkunden des Landeshauptarchivs Koblenz, Balleien Koblenz und Lothringen. Regesten (II74-1807), hrsg. v Udo Arnold, Quellen und Studien zur Geschichte des Deutschen Ordens 76 = Veröffentlichungen der Internationalen Historischen Kommission zur Erforschung des Deutschen Ordens i 4 (Weimar: Verlag und Datenbank für Geisteswissenschaften, 2016), I 7 Nr. 5 I; van Eickels, Die Deutschordensballei Koblenz, 288.

49 Regesten der Erzbischöfe, 3.2, bearb. v. Knipping, Nr. 3090; Die Deutschordensurkunden, hrsg. v. Arnold, I 7 Nr. 52.

so Die Deutschordensurkunden, hrsg. v. Arnold, 55 Nrn. 193-194. 
Freigerichte auf Zustände in Livland und Preußen. Da beide Privilegien allerdings im Koblenzer Archiv aufbewahrt wurden, ist zu vermuten, dass sie den Deutschen Orden auch am Rhein betrafen. Weitere Nachweise sind möglich. ${ }^{\text {rr }}$

Aber die Kölner Erzbischöfe waren nie ausschließliche Vollstrecker des päpstlichen Willens, auch wenn die Kurie mit dem Papst an der Spitze schon seit dem Ende des I 2. Jahrhundert zu einem übermächtigen Partner oder Gegner werden sollte. ${ }^{52}$ Erzbischof Friedrich von Saarwerden ( $1370-$ I 4 I 4) beispielsweise hat der Kurie nie die eigentlich fälligen Gebühren gezahlt. ${ }^{53}$ Dietrich von Moers ( 14 I 4- 1463) hat zwar Geld gegeben, aber er handelte in Sachen des kirchlichen Lebens als Landesherr und nicht als Erzbischof. ${ }^{54}$ Von den Nachfolgern Ruprecht von der Pfalz und Hermann von Hessen ist in dieser Hinsicht wenig zu berichten. Immerhin stellten sich der Erzbischöfe seit der zweiten Hälfte des I 4. Jahrhunderts gegen den Geldhunger der Kurie.

Die Erzbischöfe brauchten selbst Geld, vor allem um ihre landesherrlichen Aufgaben erfüllen zu können. Bei der Erstellung einer Steuer waren sie erfinderisch. Sie erhöhten die Zehnten und nahmen das subsidium caricativum ein. Es ist fraglich, wann dieses subsidium eingeführt worden sein könnte. ${ }^{55}$ Jedenfalls hatten die Kölner Erzbischöfe Einkünfte aus geistlichen Hoheitsrechten, wie der Rentmeister Johann up me Grave zu Beginn der Regierungszeit Dietrichs von Moers bilanzierte. ${ }^{56}$ Allerdings ist damals noch kein Beitrag aus dem subsidium caritatis oder dem subsidium caritativum erwähnt, jedenfalls hat der Rentmeister solche Einkünfte nicht verzeichnet. ${ }^{57}$ Auch sind der Deutsche Orden und dessen Kommenden dort nicht genannt worden. In jedem Fall hatte der Papst bereits im Jahr I 376 den Orden ausdrücklich von der Begleichung dieses subsidium befreit. ${ }^{58}$ Es

5 Die Urkunden, I, hrsg. v. Militzer, Nrn. 3 I, 38-39, 41, 322, 385 und öfter. Vgl. auch van Eickels, Die Deutschordensballei Koblenz, 209 und öfter: 208-2 I I.

52 Vgl. Feine, Kirchliche Rechtsgeschichte, 329-338.

53 Janssen, Erzbistum, I: 254-258.

54 Ebd., 27 I-276.

ss Georg Droege, Verfassung und Wirtschaft in Kurköln unter Dietrich von Moers (1414-1463), Rheinisches Archiv 50 (Bonn: Röhrscheidt, I 957), I 4 I - I 42.

s6 Droege, Verfassung, 200.

57 Vgl. Archiv für die Geschichte und Statistik des Vaterlandes, Bd. I (Bonn: Verlag des Intelligenzkomtoirs, I 785 ), i 79 .

58 Die Urkunden, II, hrsg. v. Arnold, Nr. 2346. Schon am 8. Mai I 37 I hatte der Bischof von Worms als päpstlicher Bevollmächtigter den Orden von dem Bann wegen des Subsidiums vor allem in Mainz befreit. Aber er bezog sich unter anderen auch auf die Kölner Diözese: ebd., Nr. 226I. I 419 wiederholte der Papst sein Verbot der Erhebung des Subsidiums: Die Urkunden des Deutschordenszentralarchivs in Wien. Regesten, Tlbd. III, Dezember I4I8-Dezember I526, hrsg. v. Udo Arnold, Quellen und Studien zur Geschichte des Deutschen Ordens 60/ 
muss dennoch gleichwohl im i 5 . Jahrhundert mit einem solchen subsidium gerechnet werden.

Am 20. Januar I35 I bestätigte Erzbischof Wilhelm von Gennep (I349-1362) den Komturen und den Brüdern der Deutschordenshäuser Koblenz und Köln, dass jene ihm 800 Goldgulden überreicht und dafür ihren Hof in Hermülheim bei Köln verpfändet hätten. ${ }^{59}$ Aber ansonsten ist der Kölner Erzbischof wegen der Abgaben und Steuern in den erhaltenen Urkunden nicht erwähnt.

Jedoch war gerade in den Anfängen des Deutschen Ordens besonders in Papsturkunden immer wieder über Abgaben und Steuern aller Arten die Rede. Diese Urkunden wurden vom Orden durchaus gesammelt und auch an entsprechenden Stellen vorgelegt. Dabei berücksichtigen wir nur die Bullen, die allgemein gehalten worden sind oder sich ausdrücklich auf das Deutsche Reich beziehen und Besitzungen außerhalb des Reiches im Orient, in Siebenbürgen, in Preußen oder in Livland außer Betracht lassen. ${ }^{60}$ Transsumpte und Ausstellungen von Päpsten beweisen zur Genüge die Steuererhebung von Gütern des Deutschen Ordens. Das bedeutet aber auch, dass der Deutsche Orden wegen der Abgaben- und Steuerfreiheit mit den Bischöfen und Erzbischöfen zu kämpfen hatte und dass die Bischöfe und Erzbischöfe auf ihr Besteuerungsrecht nicht ohne weiteres verzichteten. Dabei stellte der Kölner Erzbischof keine Ausnahme dar, wie wir annehmen müssen. ${ }^{61}$

Geistliche Personen, nicht nur Bischöfe oder Erzbischöfe, und auch weltliche Machthaber waren durchaus erfinderisch, wenn es galt, neue Abgaben einzuführen. Der Zehnte und die Belastung der Deutschordenshäuser durch ungebührlich hohe Einquartierungen waren nur eine von zahlreichen Möglichkeiten. Es ging um Annaten von Priester ordenseigener Pfarreien, ${ }^{62}$ den dritten oder vierten Teil von testamentarischen Übertragungen, ${ }^{63}$ den Zehnt oder Abgaben von Nah-

$/$ III = Veröffentlichungen der Internationalen Historischen Kommission zur Erforschung des Deutschen Ordens i I III (Marburg: N. G. Elwert, 2007), Nrn. 3053-3054.

s9 Die Urkunden, I, hrsg. v. Militzer, Nr. 407; Regesten der Erzbischöfe von Köln im Mittelalter, Bd. 6, I349-1362 (Wilhelm von Gennep), bearb. v. Wilhelm Janssen (Köln, Hanstein, I 977), Nr. I 2 I.

60 Tabulae, hrsg. v. Strehlke, Nrn. 306, 3 10, 31 2, 314, 316-31 7, 319 und öfter; vgl. auch oben, S. I-2.

61 Vgl. auch van Eickels, Die Deutschordensballei Koblenz, 208-2 I I.

62 Tabulae, hrsg. v. Strehlke, Nr. 327; Die Urkunden, I, hrsg. v. Arnold, Nrn. 72, 837, 967 und öfter.

63 Tabulae, hrsg. v. Strehlke, Nrn. 3 1 6, 41 3; Die Urkunden, I, hrsg. v. Arnold, Nr. 63. 
rungsmitteln, Kleidung oder Vieh, ${ }^{64}$ um einen 20. Pfennig ${ }^{65}$ oder um primitiae ${ }^{66}$. Bei weltlichen Personen handelte es sich um Steuern und Abgaben zugunsten von Burgen oder Stadtmauern. ${ }^{67}$ Alle Abgaben oder Steuern, die Geistliche oder Weltliche von Deutschordensgütern abpressen wollten, haben Päpste untersagt. Ob den Aufforderungen stets gehorcht wurde, mag dahin gestellt bleiben. Immerhin deutet die oftmalige Wiederholung solcher Verbote auf eine Nichteinhaltung hin.

Der Ritt nach Rom und an die Kurie, auch wenn sie in Avignon war, blieb beschwerlich und wurde noch beschwerlicher, wenn ein Mann zu Fuß den Weg hin und zurück machte. Es war auf jeden Fall einfacher und auch preiswerter, wenn man sich in der Umgebung einen Urteiler oder Schiedsrichter suchte. So beauftragte beispielsweise der Kölner Erzbischof Walram von Jülich den Dekan von St. Georg um die Schlichtung einer Auseinandersetzung wegen der Pfarre von Neurath. Der Dekan wiederum betraute Pfarrer mit der Auseinandersetzung. ${ }^{68}$ Allerdings war der Dekan von St. Georg in Köln schon ein vom Papst ernannter Konservator des Ordens. Vor dem Dekan von St. Florin in Koblenz klagte im Jahr I 259 ein Beauftragter des Komturs von Koblenz gegen Dietrich von Brachelen wegen eines Hofs in der Pfarrei Güsten östlich von Jülich. Der Dekan war vom Kölner Erzbischof als Konservator der Rechte des Ordens eingesetzt worden. Den Erzbischof selbst hatte der Papst ernannt. ${ }^{69}$ Die Liste lässt sich ohne weiteres fortsetzen, ohne dass allerdings immer Erzbischöfe oder Bischöfe als Urteiler oder Schiedsrichter in Frage gekommen wären.

Im I 4. und zunehmend im I 5 . Jahrhundert haben Kölner Erzbischöfe immer wieder in die Richtlinienkompetenz der Päpste eingegriffen. Die Päpste ihrerseits ernannten Prälaten vor Ort, um Streitfälle zu entscheiden, oder auch Konservatoren der Rechte des Deutschen Ordens. Besonders seit dem Papstjahren Martins V. ( I 4I 7-I 43 I) ist das zu beobachten. Allerdings ist auch anzumerken, dass die Ritterorden keine herausragende Rolle im Rheinland oder in der Erzdiözese Kölns spielten..$^{7}$ Immerhin mögen die drei genannten Einfallstore die Eingliederung in

${ }^{64}$ Tabulae, hrsg. v. Strehlke, Nr. 330; Die Urkunden, I, hrsg. v. Arnold, Nrn. 74, I 72, I 75, 376, 43 I und öfter.

65 Tabulae, hrsg. v. Strehlke, Nr. 332; Die Urkunden, I, hrsg. v. Arnold, Nrn. 78, 279, 325.

66 Tabulae, hrsg. v. Strehlke, Nr. 430; Die Urkunden, I, hrsg. v. Arnold, Nrn. 28, 170 und öfter.

67 Tabulae, hrsg. v. Strehlke, Nr. 345; Die Urkunden, I, hrsg. v. Arnold, Nrn. 85, 287.

68 Die Urkunden, I, hrsg. v. Militzer, Nrn. 385, 387; Regesten der Erzbischöfe von Köln im Mittelalter, Bd. 5, I332-I349 (Walram von Jülich), bearb. v. Wilhelm Janssen (Düsseldorf-Bonn, Droste, Hanstein, I 973 ), Nr. I 3 I 0.

69 Die Urkunden, I, hrsg. v. Militzer, Nr. 443; Regesten der Erzbischöfe, 6, bearb. v. Janssen, Nr. 1263.

70 Janssen, Erzbistum, I: 576-577. 
den Diözesanverband des Kölner Erzbistums erleichtert haben, ohne dass ich andere Möglichkeiten ausschließen möchte oder könnte, da es sie durchaus gab.

\section{PRIMARY SOURCES:}

Codex diplomaticus ordinis sanctae Mariae Theutonicorum. Urkundenbuch des Deutschen Ordens. Bd. 2. Herausgegeben von Johannes Heinrich Hennes. Mainz: Schott und Thielmann Kirchheim, I845-186r.

Die Deutschordensurkunden des Landeshauptarchivs Koblenz, Balleien Koblenz und Lothringen. Regesten (II74-I807). Herausgegeben von Udo Arnold. Quellen und Studien zur Geschichte des Deutschen Ordens $76=$ Veröffentlichungen der Internationalen Historischen Kommission zur Erforschung des Deutschen Ordens I 4. Weimar: Verlag und Datenbank für Geisteswissenschaften, 2016.

Die Urkunden der Deutschordenskommende St. Katharinen zu Köln. Regesten. Tlbd. I, Juni I218 - November I496. Herausgegeben von Klaus Militzer. Quellen und Studien zur Geschichte des Deutschen Ordens 78/I = Veröffentlichungen der Internationalen Historischen Kommission zur Erforschung des Deutschen Ordens 16/I. Weimar: Verlag und Datenbank für Geisteswissenschaften, 20 I 6.

Die Urkunden des Deutschordenszentralarchivs in Wien. Regesten. Tlbd. I, II22 - Januar I313. Herausgegeben von Udo Arnold. Quellen und Studien zur Geschichte des Deutschen Ordens 60/I = Veröffentlichungen der Internationalen Historischen Kommission zur Erforschung des Deutschen Ordens i I/I. Marburg: N. G. Elwert, 2006).

Die Urkunden des Deutschordenszentralarchivs in Wien. Regesten. Tlbd. II, Februar 1313-November 14I8. Herausgegeben von Udo Arnold. Quellen und Studien zur Geschichte des Deutschen Ordens 60/II = Veröffentlichungen der Internationalen Historischen Kommission zur Erforschung des Deutschen Ordens I I/II. Marburg: N. G. Elwert, 2007.

Die Urkunden des Deutschordenszentralarchivs in Wien. Regesten. Tlbd. III, Dezember I4I8 - Dezember I526. Herausgegeben von Udo Arnold. Quellen und Studien zur Geschichte des Deutschen Ordens 60/III = Veröffentlichungen der Internationalen Historischen Kommission zur Erforschung des Deutschen Ordens I I/III. Marburg: N. G. Elwert, 2007.

Urkundenbuch für die Geschichte des Niederrheins oder des Erzstifts Cöln, der Fürstenthümer Jülich und Berg, Geldern, Meurs, Cleve und Mark, und der Reichsstifte Elten, Essen und Werden. Bd. 2, Von dem Jahr I20I bis I30o einschliesslich. Herausgegeben von Theodor Joseph Lacomblet. Düsseldorf: J. Wolf, I 846.

Regesta historico-diplomatica Ordinis S. Mariae Theutonicorum. Tl. I, Index Tabularii Ordinis S. Mariae Theutonicorum / Regesten zum Ordensbriefarchiv. Bearbeitet unter Mitwirkung zahlreicher Anderer v. Erich Joachim. Heausgegeben von Walther Hubatsch, Bd. 2, I455-1510. Göttingen: Vandenhoeck \& Ruprecht, 1950. 
Regesten der Erzbischöfe von Köln im Mittelalter. Bd. 3, I205-I304, Hälfte I, I205-I26I.

Bearbeitet von Richard Knipping. Düsseldorf-Bonn: Droste, Hanstein, I 909.

Regesten der Erzbischöfe von Köln im Mittelalter. Bd. 3, I205-I304, Hälfte 2, I26I-I304

und Register zu beiden Hälften. Bearbeitet von Richard Knipping. Düsseldorf-Bonn:

Droste, Hanstein, I9 13.

Regesten der Erzbischöfe von Köln im Mittelalter. Bd. 4, I304-I332. Bearbeitet von Wil-

helm Kisky. Düsseldorf-Bonn: Droste, Hanstein, i 9 I 5 .

Regesten der Erzbischöfe von Köln im Mittelalter. Bd. 5, I332-I349 (Walram von Jülich).

Bearbeitet von Wilhelm Janssen. Düsseldorf-Bonn, Droste, Hanstein, I 973.

Regesten der Erzbischöfe von Köln im Mittelalter. Bd. 6, I349-1362 (Wilhelm von Gennep).

Bearbeitet von Wilhelm Janssen. Köln, Hanstein, 1977.

Regesten der Erzbischöfe von Köln im Mittelalter. Bd. 9, I38I-I390 (Friedrich von Saarwerden). Bearbeitet von Norbert Andernach. Düsseldorf: Droste, I 983.

Tabulae Ordinis Theutonici. Herausgegeben von Ernst Strehlke. Berlin: Weidmann, I 869.

Neudruck: Toronto: Prelum Academicum Universitatis Torontonensis, I 975.

\section{SECONDARY SOURCES:}

Arnold, Udo. “Der Deutsche Orden zwischen Kaiser und Papst im I 3. Jahrhundert." In Ritterorden zwischen geistlicher und weltlicher Macht, herausgegeben von Zenon $\mathrm{Hu}-$ bert Nowak. Ordines Militares. Colloquia Torunensia Historica V, 57-70. Toruń: Wydawnictwo Uniwersytetu Mikołaja Kopernika, 990.

Droege, Georg. Verfassung und Wirtschaft in Kurköln unter Dietrich von Moers (I4I4-1463). Rheinisches Archiv 50. Bonn: Röhrscheidt, I 957.

Eickels van, Klaus. Die Deutschordensballei Koblenz und ibre wirtschaftliche Entwicklung im Spätmittelalter. Quellen und Studien zur Geschichte des Deutschen Ordens 52. Marburg: N. G. Elwert, I 995.

Favreau-Lilie, Marie-Luise. "Reformbedarf und Reformen an der norditalienischen Peripherie. Der Deutsche Orden in Venedig und in Venetien (14.-1 5. Jh.)," Ordines Militares Colloquia Torunensia Historica. Yearbook for the Study of the Military Orders I 6 (20 I I): 6I -85

Feine, Hans Erich. Kirchliche Rechtsgeschichte. Katholische Kirche. Köln-Graz: Böhlau, 1964.

Gescher, Franz. "Das Offizialat der Erzbischöfe von Köln im I 3. Jahrhundert." Annalen des Historischen Vereins für den Niederrhein i I 5 ( 1 929): I $36-166$.

Gescher, Franz. "Die erzbischöfliche Kurie von ihren ersten Anfängen bis zur Gegenwart, Eine rechtsgeschichtliche Skizze." Annalen des Historischen Vereins für den Niederrhein I I 8 ( I 93 I): I-3 I.

Janssen, Wilhelm. "Bemerkungen zum Aufkommen der Schiedsgerichtsbarkeit am Niederrhein im I 3. Jahrhundert." Jahrbuch des Kölnischen Geschichtsvereins 43, H. I (197 I): 77- I00.

Janssen, Wilhelm. Das Erzbistum Köln im späten Mittelalter, IIgI-ISIS. Tl. I. Geschichte des Erzbistums Köln 2. Köln: Bachem, I 995. 
Limburg, Hans. Die Hochmeister des Deutschen Ordens und die Ballei Koblenz. Quellen und Studien zur Geschichte des Deutschen Ordens 8. Bad Godesberg: Verlag Wissenschaftliches Archiv, 1969.

Militzer, Klaus. "Die Einbindung des Deutschen Ordens in die süddeutsche Adelswelt," In Ritterorden und Region. Politische, soziale und wirtschaftliche Verbindungen im Mittelalter, herausgegeben von Zenon Hubert Nowak. Ordines Militares. Colloquia Torunensia Historica VIII, 14I-160. Toruń: Wydawnictwo Uniwersytetu Mikołaja Kopernika, 1995. Wiederabdruck In Klaus Militzer, Zentrale und Region. Gesammelte Beiträge zur Geschichte des Deutschen Ordens in Preussen, Livland und im Deutschen Reich aus den Jahren I968 bis 2008. Quellen und Studien zur Geschichte des Deutschen Ordens 75 = Veröffentlichungen der Internationalen Historischen Kommission zur Erforschung des Deutschen Ordens I 3, 322-34I. Weimar: vdg, 20 I 5.

Militzer, Klaus. Von Akkon zur Marienburg. Quellen und Studien zur Geschichte des Deutschen Ordens 56 = Veröffentlichungen der Internationalen Historischen Kommission zur Erforschung des Deutschen Ordens 9. Marburg: N. G. Elwert, 1999.

Toomaspoeg, Kristjan. "Der Verlust der Besitzungen des Deutschen Ordens in Italien am Ende des I 5 . und zu Beginn des I6. Jahrhunderts." Ordines Militares Colloquia Torunensia Historica. Yearbook for the Study of the Military Orders I6 (201 I): I 29-I 54. 